\title{
Tantalum Pentoxide Waveguides and Microresonators for VECSEL-based Frequency Combs
}

\author{
T. Chen Sverre , J. R. C. Woods ${ }^{\mathrm{a}}$, E. A. Shaw ${ }^{\mathrm{a}}$, Ping Hua ${ }^{\mathrm{b}}$, V. Apostoulopoulos ${ }^{\mathrm{a}}$, J. S. \\ Wilkinson $^{\mathrm{b}}$, and A. C. Tropper ${ }^{\mathrm{a}}$ \\ ${ }^{a}$ Department of Physics and Astronomy, University of Southampton, Highfield Road \\ Southampton, United Kngdom, SO17 1BJ \\ ${ }^{\mathrm{b}}$ Optoelectronics Research Centre, University of Southampton, Highfield Road Southampton, \\ United Kngdom, SO17 1BJ
}

\begin{abstract}
Tantalum pentoxide $\left(\mathrm{Ta}_{2} \mathrm{O}_{5}\right)$ is a promising material for mass-producible, multi-functional, integrated photonics circuits on silicon, exhibiting robust electrical, mechanical and thermal properties, as well as good CMOS compatibility. In addition, $\mathrm{Ta}_{2} \mathrm{O}_{5}$ has been reported to demonstrate a non-linear response comparable to that of chalcogenide glass, in the region of 3-6 times larger than that of materials such as silica $\left(\mathrm{SiO}_{2}\right)$ or silicon nitride $\left(\mathrm{Si}_{3} \mathrm{~N}_{4}\right)$. In contrast to Si-based dielectrics, it will accept trivalent ytterbium and erbium dopant ions, opening the possibility of on-chip amplification. The high refractive index of $\mathrm{Ta}_{2} \mathrm{O}_{5}$ is consistent with small guided mode cross-section area, and allows the construction of micro-ring resonators. Propagation losses as low as 0.2 $d B / \mathrm{cm}$ have been reported. In this paper we describe the design of a planar $\mathrm{Ta}_{2} \mathrm{O}_{5}$ waveguides optimised for the generation of coherent continuum with near infrared pulse trains at $\mathrm{kW}$ peak powers. The Pulse Repetition Frequency (PRF) of the VECSEL can be tuned to a sub-harmonic of the planar micro-ring and the optical pump power applied to the VECSEL can be adjusted so that mode-matching of the VECSEL pulse train with the micro-ring resonator can be achieved. We shall describe the fabrication of $\mathrm{Ta}_{2} \mathrm{O}_{5}$ guiding structures, and the characterisation of their nonlinear and other optical properties. Characterisation with conventional lasers will be used to assess the degree of coherent spectral broadening likely to be achievable using these devices when driven by mode-locked VECSELs operating near the current state-of- art for pulse energy and duration.
\end{abstract}

Keywords: VECSEL, Mode-locked, Frequency Combs, Supercontinuum

\section{INTRODUCTION}

Broad wavelength laser emission has been of use for a large number of fields, and as the power, repetition frequency and breadth increase, even more techniques and technologies become available. Non-linear processes have been harnessed extensively in optical systems to provide broadband wavelength sources capable of high power spectroscopy, and with sufficient coherence for metrology. Frequency combs, consisting of individual equidistant optical frequency components, have been of keen interest to the scientific community as a whole ${ }^{1}$, even attaining the Nobel Prize in 2005. A frequency comb is of such importance because it can be used as an optical ruler, measuring unknown frequencies by beating them against the comb. This can be applied with great accuracy as long as an optical component can be referenced to an absolute frequency value, that is stabilising the Carrier Envelope Offset frequency $\left(f_{C E O}\right)$. An effective way of achieving this is to produce an octavespanning comb, so that the lowest frequency component can be doubled and compared to the highest frequency component, in an "f-to-2f measurement". Unfortunately, non-linear processes require intense pulses with peak powers exceeding $\mathrm{kW}$ to drive a broad continuum at sufficient power; as a result, a typical supercontinuum system will be based around a high power mode-locked laser, e.g. Ti:Sapphire or MOPA system. Due to their high peak power and pulse durations, these lasers can efficiently use the second order refractive index in silica $\left(n_{2}=2.6 \mathrm{e}-20 \mathrm{~m}^{2} / W^{2}\right)$, which is low loss at telecom wavelengths, readily available and easy to manufacture.

Further author information: (Send correspondence to T. Chen Sverre)

T. Chen Sverre: E-mail: t.chen-sverre@soton.ac.uk, Telephone: +44(0)2380592066 
Frequency combs of $\mathrm{GHz}$ repetition rates have been sparsely demonstrated by using microresonators and mode filtering ${ }^{3}$, and remain a desirable objective. The mode spacing of a $1-100 \mathrm{GHz}$ frequency comb is sufficient to resolve individual modes with a spectrometer making the comb much more easily applied. To produce a frequency comb of this spacing, a $\mathrm{GHz}$ microresonator can be driven with a $\mathrm{CW}$ laser, leading to continuum generation via parametric amplification in materials with high $n_{2}$. Thanks to their cavity lengths and carrier lifetimes, VECSELs typically produce approximately $0.5-5 \mathrm{GHz}$ pulse trains (though they can span a broader range), therefore Pulse Repetition Frequency (PRF) can be tuned to drive a microresonator at a sub-harmonic for enhanced amplification. The ability to produce a resonantly enhanced solution to GHz frequency combs, from a compact laser source and target, will enable applications in spectroscopy, optical arbitrary waveform generation, astrophysical spectrometer calibration and telecommunications, which require frequency combs with large mode spacing ${ }^{4}$.

\section{VECSEL PERFORMANCE}

VECSELs, also known as Semiconductor Disk Lasers (SDLs), have gained a great deal of interest in recent years both in CW and ML operation. Semiconductor material may be grown to provide emission at a variety of wavelengths, for narrow linewidth or mode-locking. Over the last few years refinements to the growth process and design of gain structures has lead to pulse duration and output power records approaching the performance of the highest performance mode-locked lasers. To enable fundamental mode-locking, VECSELs are typically built with a Semiconductor Saturable Absorbing Mirror (SESAM). SESAMs must be grown to precise specifications to pair properly with a given VECSEL gain structure. As a whole, the VECSEL structure pair must be designed to balance GDD, non-saturable losses, modulation depth, or transform-limited pulses may become inaccessible at high powers. Since both VECSEL gain structures and SESAMs can both be fabricated in the same process Mode-locked Integrated eXternal-cavity Surface Emitting Lasers (MIXSELs) have been developed to combine and active region and saturable absorber into a single semiconductor structure. Finally, Self Mode Locking (SML) has been reported for VECSELs but has yet to generate sub 500-fs pulse durations and must be researched further.

Design of VECSEL structures is critical to reduction in pulse durations with average powers near 1-W. Dispersion management through careful microcavity design and dielectric coating has resulted in huge advances in ML performance. In addition, thermal management in VECSELs has advanced significantly in recent years, which has vastly improved the power handling capabilities of VECSEL structures. "Flip-chip" design and acid etching of substrates has eliminated the issues of thermal extraction through large amounts of semiconductor material. This can be coupled with intra- or extra-cavity heat-spreaders, which are becoming a standard feature of high performance results. The most noteworthy variant of thermally managed VECSELs is the Membrane External Cavity Surface Emitting Laser (MECSEL) ${ }^{34-36}$ where the periodic gain structure is acid-etched completely from

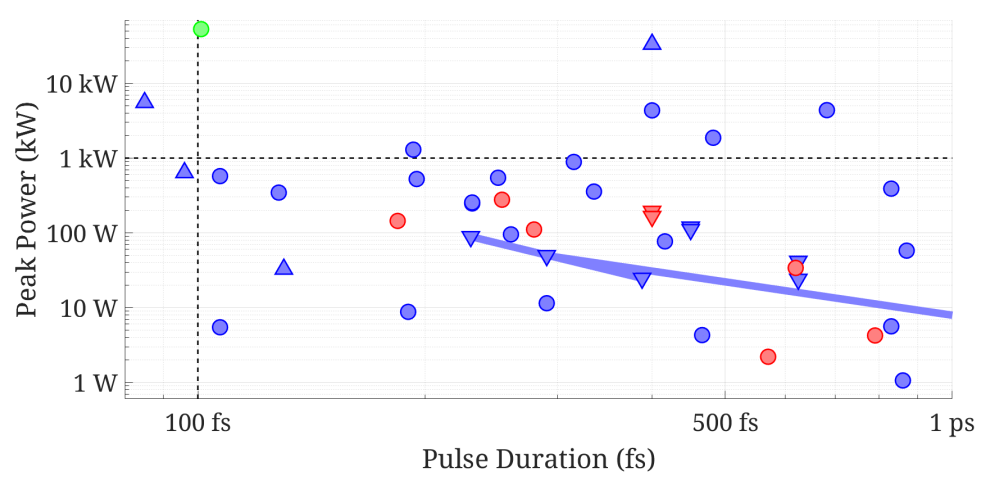

Figure 1. (On a log-log scale) Peak power results for sub-ps VECSELs (blue) $)^{5-27}$ and MIXSELs (red) ${ }^{28-33}$. Results are distinguished by direct output (circle) external pulse compression (upward-triangle) and repetition-rate tunable (downwardtriangle). The peak power and pulse duration of the ML Ti:Sapphire laser used in this paper (Fig.3) is also displayed (green). Two dashed lines delimit $1 \mathrm{~kW}$ peak power and 100-fs pulse duration respectively. 
the substrate in the absence of a DBR to produce a membrane of QWs which is suspended between heatspreaders. All efforts to improve the thermal management of the gain structure are instrumental to pushing ML-VECSEL output into the multi-Watt regime.

Compared to conventional systems, VECSELs have only recently been capable of exceeding 1-kW peak powers at pulse durations below $200 \mathrm{fs}^{23}$, and even more recently approaching $1 \mathrm{~kW}$ at pulse durations of less than 100 $\mathrm{fs}^{8}$. A further restriction to peak power is the high pulse PRF typically exceeding $1 \mathrm{GHz}$. In addition, power scaling and emission wavelength are dependent on the semiconductor growth, and may not necessarily align with conventional continuum components (Ti:Sapphire or telecom wavelengths). Therefore, careful design of both the VECSEL and the target material is necessary to match the performance of the standard frequency-comb solutions. Fig.1 displays the current state of the field for fundamental mode-locking for VECSELs and MIXSELs (from $50 \mathrm{fs}-1 \mathrm{ps}$, and $0.1 \mathrm{~W}-10 \mathrm{~kW}$ ). Notable results are at pulse durations of less than $100 \mathrm{fs}^{8}$, and higher peak powers than $1 \mathrm{~kW}^{14,23}$.

Previous work on VECSEL-continuum has been performed by Wilcox et al. (2013) ${ }^{14}$, using a thermally managed ML-VECSEL producing $400 \mathrm{fs}$ coupled into $2 \mathrm{~m}$ of $2.2-\mu \mathrm{m}$ core Photonic Crystal Fibre (PCF). At a coupled power of $0.5 \mathrm{~W}(\approx 0.75 \mathrm{~kW})$ the output spectrum was up to $175 \mathrm{~nm}$. Head et.al. $(2013)^{22}$, also reported supercontinuum of up to $280 \mathrm{~nm}$ while pumping $1 \mathrm{~m}$ of of 4.8- $\mu \mathrm{m}$ core PCF. The VECSEL output was externally amplified and compressed (VECSEL-MOPA) resulting in $40 \mathrm{~W}$ of 400-fs pulses, at $1040 \mathrm{~nm}$, and a $\mathrm{PRF}$ of 3 -GHz. The PCF average output power was $2.5 \mathrm{~W}$, equivalent to a minimum coupled peak power of $\approx 2 \mathrm{~kW}$. These results were primarily limited by the power handling of the PCFs. The average power required to drive supercontinuum at 400fs and PRF of $1.7 \mathrm{GHz}$ and $3 \mathrm{GHz}$ respectively, is close to the PCF damage threshold. Improving pulse durations and average powers may lead to significant improvements in broadening results, however, this will lead to an proportionally higher average power. Therefore, broad continuum generation will rely on improving power handling and if possible reducing required peak power of target structures. These requirements can be addressed by using materials with high $n_{2}$, and microstructure development for resonant enhancement.

\section{NOVEL MATERIALS FOR NON-LINEAR GENERATION}

Silica $\left(\mathrm{SiO}_{2}\right)$ has become a standard material for comb generation, recent developments into on-chip materials and chalcogenides have demonstrated low coupling and propagation loss, and much higher $n_{2}$ critical for compensating for lower peak power laser sources. In particular, Tantalum Pentoxide $\left(\mathrm{Ta}_{2} \mathrm{O}_{5}\right)$ has been demonstrated to have propagation losses of approximately $0.2 \mathrm{~dB} / \mathrm{cm}$ have been reported and an $n_{2} 3-6.5$ times larger than $\mathrm{Si}_{3} \mathrm{~N}_{4}$ and $\mathrm{SiO}_{2}{ }^{38}$. Tantalum Pentoxide is also CMOS compatible, opening up the possibility of on-chip fabrication, and microstructuring, pairing well with the GHz PRF of VECSELs to pump a microstructure on-resonance.

(a)

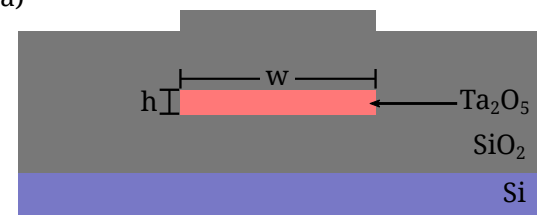

(c)

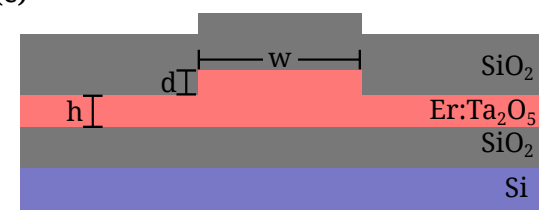

(b)

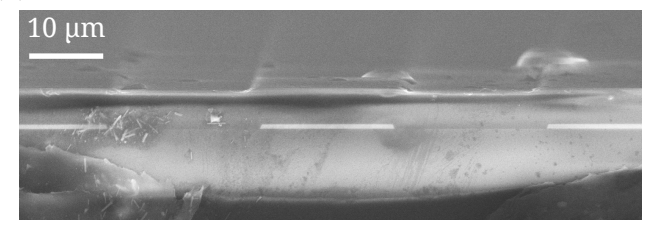

(d)

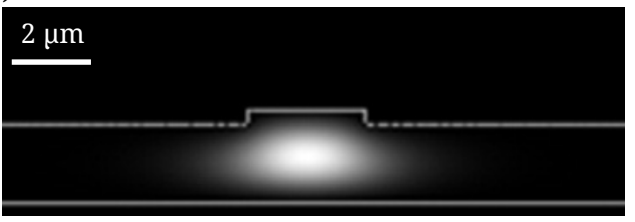

Figure 2. (a) A schematic diagram of a strip waveguide. The waveguides are composed of a Si wafer, coated with a layer of $\mathrm{SiO}_{2}$, a Ta $\mathrm{Ta}_{5}$ active region and $\mathrm{SiO}_{2}$ cladding on top. (b) An SEM image of a strip waveguide input cross-section (c) A schematic diagram of a rib waveguide composed of Er-doped $\mathrm{Ta}_{2} \mathrm{O}_{5}$. (d) Theoretical mode shape of a $\mathrm{W}=2 \mu \mathrm{m}$ rib waveguide $^{37}$. 

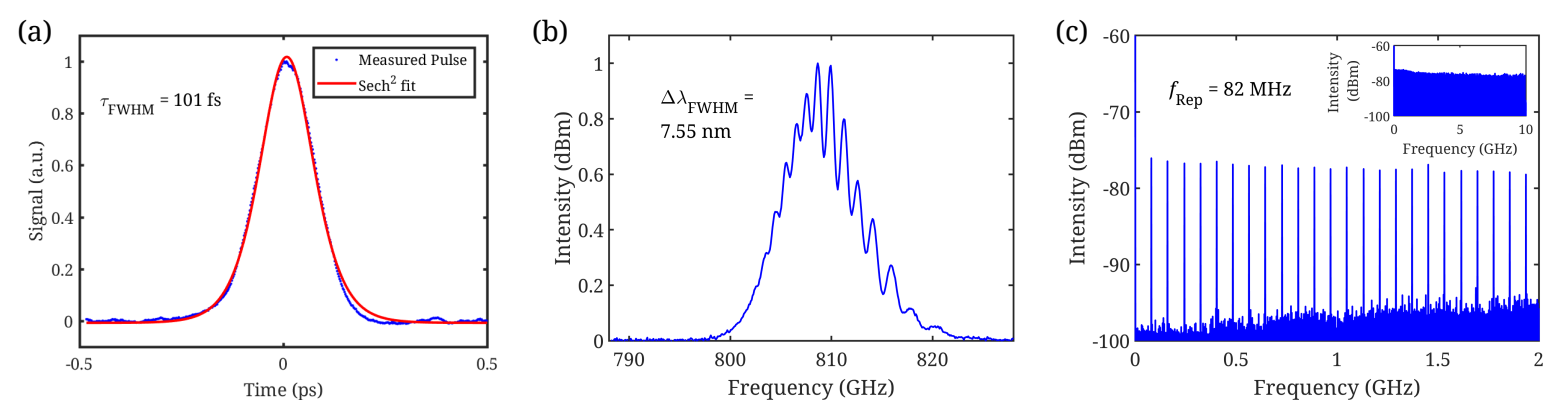

Figure 3. Mode-locked characterisation of the ML-Ti:Sapphire used to drive waveguides. (a) Intensity autocorrelation with a sech ${ }^{2}$ fit retrieving a pulse duration of $101 \mathrm{fs}$. (b) Optical spectrum displays a centre wavelength of $808 \mathrm{~nm}$ and a FWHM of approximately $7.55 \mathrm{~nm}$ ( $\approx 1.1$ times bandwidth limit). (c) RF spectrum showing a PRF of $82 \mathrm{MHz}$.

$\mathrm{Ta}_{2} \mathrm{O}_{5}$ has been heralded as an ideally suited material for photonic devices and circuits. It exhibits a number of properties that make it an attractive medium for non-linear generation. It is transparent from $150 \mathrm{~nm}$ up to 4 $\mu \mathrm{m}$ with reported propagation losses of approximately $2 \mathrm{~dB} / \mathrm{cm}$; this presents a major advantage over semiconductor materials which typically have limited transparency windows and are thus unsuitable for passive devices. Perhaps most important, is that the third-order ( $\chi^{3}$ effects) are comparable to chalcogenides, $n_{2}=7.23 \mathrm{e}-19$ $m^{2} / W$, significantly higher than $\operatorname{SiN}\left(n_{2}=2.3 \mathrm{e}-19 \mathrm{~m}^{2} / W\right) \cdot \mathrm{Ta}_{2} \mathrm{O}_{5}$ also benefits from compatibility with CMOS processing and can be sputtered onto Silicon ( $\mathrm{Si}$ ) and for lower losses techniques such as Selective Oxidation of Refractory Material (SORM) have been applied to refine the structures. $\mathrm{SORM}$ is a process that uses a $\mathrm{SiO}_{2}$ mask and e-beam to selectively uncover sputtered $\mathrm{Ta}_{2} \mathrm{O}_{5}$ for precise and high quality waveguide and microstructure fabrication reducing propagation losses compared to standard techniques ${ }^{39,40}$. This process allows highly tunable design of high index waveguides, allowing zero dispersion wavelength (ZDW) and microresonators to be optimised. Further improvements over other materials include the possibility of direct grating couplers which can greatly improve performance over high confinement fluoride glasses. Fluoride glasses (including ZBLAN), have demonstrated very high non-linear response $\left(n_{2}=5.4 \mathrm{e}-16 \mathrm{~m}^{2} / W\right)$ producing $200-2,500 \mathrm{~nm}$ with nJ pulses of 1042-nm light ${ }^{41}$. Unfortunately, these structures with high confinement typically have to balance the advantages fo confinement with high coupling and propagation losses.

For this research we have obtained two sets of waveguides, strip and rib, composed of $\mathrm{Ta}_{2} \mathrm{O}_{5}$ and $\mathrm{Er}_{2}$ Ta $2 \mathrm{O}_{5}$ respectively. Both were designed for a pump wavelength close to $1.5 \mu \mathrm{m}$ but will serve as a first step toward targeted waveguide design for 1- $\mu \mathrm{m}$. To test the performance of these waveguides we built a setup based on a ML-Ti:Sapphire laser emitting a pulse train of $\approx 100-\mathrm{fs}$ pulses at $808 \mathrm{~nm}$, at a PRF of $82 \mathrm{MHz}$ and with an average power of $470 \mathrm{~mW}$, approximately $53 \mathrm{~kW}$ peak power (Fig.3). The ends of the waveguides are polished and the NA of the waveguides is approximately 0.5-0.75, to match this condition the closest focussing element available fo our experimentation was an aspheric of $\mathrm{NA}=0.44$. Power was recorded using a PhotoDiode (PD), and both the output port of the waveguide and lateral emission (in the Er-doped case) were analysed with OSAs.

The first set of waveguides were $5 \mathrm{~mm}$ long strip waveguides $0.6 \mu \mathrm{m}$ high and $20 \mu \mathrm{m}$ wide. The large width versus height difference imposes a strong preference on horizontal polarisation. Fig.2(b) displays the increase in spectral width for $53 \mathrm{~kW}$ incident on the input port, resulting in an increase from $\approx 25 \mathrm{~nm}$ to $\approx 60 \mathrm{~nm}($ at $-20 \mathrm{dBm} / \mathrm{nm})$. As this was performed on a very small propagation length, with high insertion and propagation losses, this result indicates the potential of even very small sections of $\mathrm{Ta}_{2} \mathrm{O}_{5}$.

The second set are waveguides used in Subramanian et al. (2010) ${ }^{42}$. These waveguides are 2-cm long, Ta $2 \mathrm{O}_{5}$ doped with Er, at a concentration of $2.7 \mathrm{e} 20$ ions $/ \mathrm{cm}^{3}$, in a rib structure (shown in Fig.2(c)). The rib design has been selected and optimised to produce a larger mode than a strip waveguide, with lower reflection losses associated with propagation. Fig.2(b and c) display the output port andlateral emission of the waveguides as a function of peak power. The waveguide output port was used to collect the IR mode, but the laterally emitted green (550-nm) was not strongly guided and was collected by a multimode fibre in close proximity to the waveguide surface. The low confinement/larger mode in these rib waveguides suppresses nonlinear generation and the broadening of the output port spectrum. In addition, the high concentration of Er strongly absorbs the mode power. The waveguide displays lateral emission of 540-nm light with a FWHM of approximately 40 
(a)

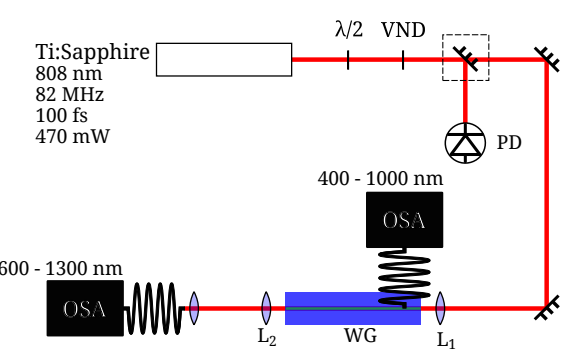

(c)

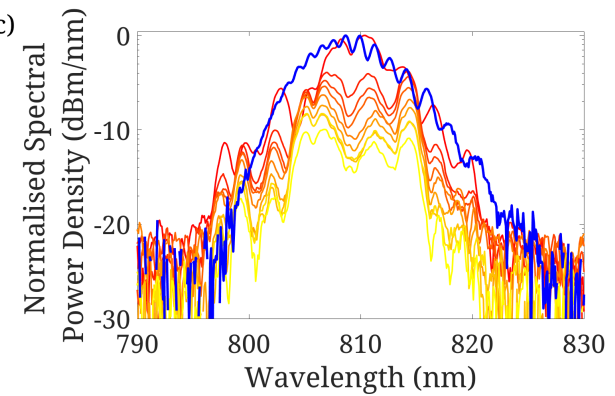

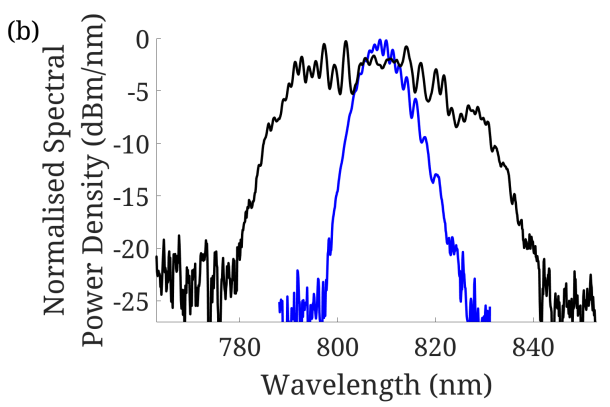

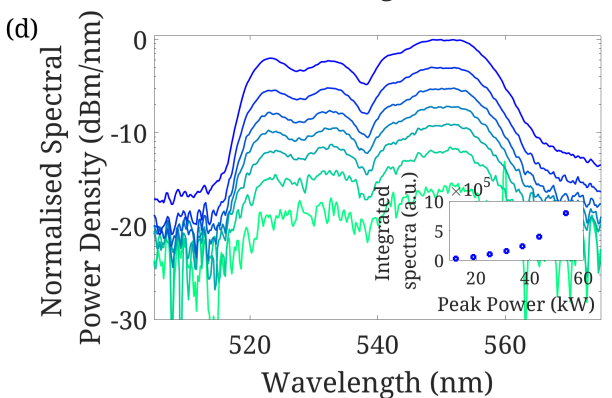

Figure 4. (a) Experimental setup used for testing the waveguides under high peak-power pulse trains. The output of a ML-Ti:Sapphire laser was coupled into the waveguide using a lens of $N A=0.44$, and collected on exit from the guide by a lens of $\mathrm{NA}=0.4$. (b) The output from the $\mathrm{Ta}_{2} \mathrm{O}_{5}$ waveguide (black). (c) The output from the Er:Ta $\mathrm{O}_{5}$ waveguide. Both (b) and (c) are displayed as a function of launched peak power $12.5-53.5 \mathrm{~kW}$, and compared to the input spectrum Fig.3(b). (d) The green emission from the Er:Ta $\mathrm{O}_{5}$, (inset) the retrieved spectral density for the lateral emission is also displayed (inset).

$\mathrm{nm}$, which has previously been attributed to parametric amplification ${ }^{?}$, and Er: $\mathrm{Ta}_{2} \mathrm{O}_{5} \mathrm{PL}^{43}$ via two photon absorption. The pumping of this emission is performed at $808 \mathrm{~nm}$ and retrieval of spectral density displays a power relationship of $2.6 \pm 0.2$, consistent with the expectation of square law for both processes.

The waveguide performance shown here displays the potential for low loss modes in $\mathrm{Ta}_{2} \mathrm{O}_{5}$ waveguides, successfully guiding highly energetic pulse trains. $\mathrm{Ta}_{2} \mathrm{O}_{5}$ strip waveguides demonstrated an increase of $25 \mathrm{~nm}$ to $60 \mathrm{~nm}$ over a $5 \mathrm{~mm}$ section without thermal management, fully optimised coupling optics or rib structure

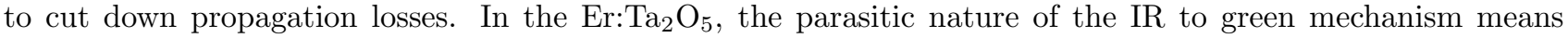
that these waveguides are unsuitable to exploit the $\mathrm{n}_{2}$ of $\mathrm{Ta}_{2} \mathrm{O}_{5}$ but will be used to design further waveguides for low loss at our VECSEL wavelength. Furthermore, the peak power available with a Ti:Sapphire laser is at least an order of magnitude higher than that currently achievable with a VECSEL, and pulse durations of $<100$ fs are only just becoming available to VECSEL research. External amplification and pulse compression may lead to significant increases in VECSEL generated continuum, however the huge average power required to match conventional laser peak power will pose an obstacle. As such, the route to efficient use of $\mathrm{Ta}_{2} \mathrm{O}_{5}$ with the current generation of VECSELs, is to exploit the VECSEL PRF and $\mathrm{Ta}_{2} \mathrm{O}_{5}$ fabrication methods for resonant enhancement of microstructures.

Microresonators have been demonstrated for telecom wavelengths in a variety of materials, typically in $\mathrm{Si}_{3} \mathrm{~N}_{4}$ due to its CMOS compatibility ${ }^{44}$, a property $\mathrm{Ta}_{2} \mathrm{O}_{5}$ shares. Rabiei et al. $(2014)^{38}$ have reported $\mathrm{Ta}_{2} \mathrm{O}_{5}$ microresonators of $200-300 \mu \mathrm{m}$ diameter with propagation losses lower than $4.9 \mathrm{~dB}$ and $\mathrm{Q}$ factors higher than $10^{5}$. Resonators of this quality and material are of great interest to VECSELs and non-linear generation as a whole, and they even provide a route to on-chip solutions and devices. In addition, simulations performed by Rabiei et al. of Grating Couplers (GC) indicate that possible coupling losses may be as low as $2 \mathrm{~dB}$, drastically improving coupled peak power and reducing the potential damage to the waveguide under a VECSEL's average power.

To develop and optimise $\mathrm{Ta}_{2} \mathrm{O}_{5}$ microresonator, the properties of traditional rib waveguides must first be investigated to determine zero-dispersion designs, and minimise coupling and propagation losses at VECSEL 


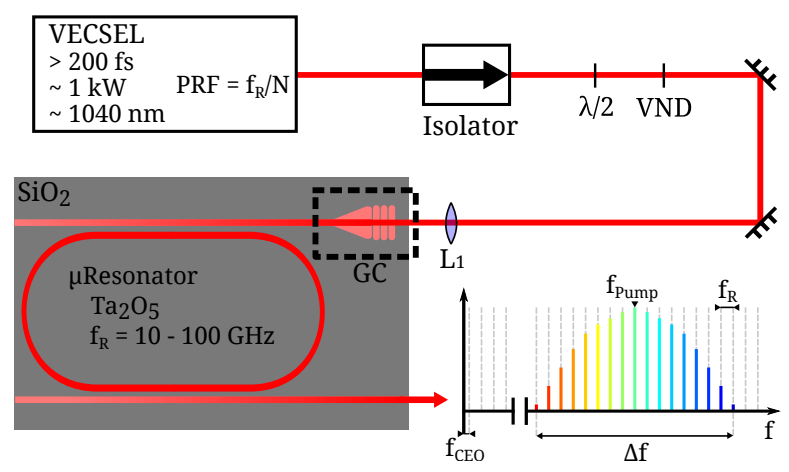

Figure 5. A schematic diagram of a ML-VECSEL punping a microresonator ( $\mu$ Resonator) for continuum generation. The VECSEL will be tuned to the Nth subharmonic of the microresonator and the output will be isolated. Power, polarisation and coupling will be controlled via Variable ND filters (VND), a half wave plate $(\lambda / 2)$ and an $N A$ selected objective $\left(L_{1}\right)$. The waveguide coupling can be further optimised for high power with a Grating Coupler (GC). The microresonator $f_{R}$ will be of the order $10-100 \mathrm{GHz}$ to target a large mode spacing frequency comb. As long as the span of the comb $\Delta f$ is broad enough the $f_{C E O}$ can be stabilised for a coherent and absolute frequency measurement.

wavelengths. Fig.5 displays a proposed configuration of a ML-VECSEL to produce coherent supercontinuum. A VECSEL with PRF tuned to the Nth subharmonic of a microresonator $\left(\mathrm{f}_{R}\right)$ will be coupled into a waveguide, directly or via a grating coupler (GC). To optimise the performance of the system, the VECSEL must be isolated, to reduce destabilising backreflections, and power and polarisation must be controlled, with VND and $\lambda / 2$. The resonator itself will be based on $\mathrm{Ta}_{2} \mathrm{O}_{5}$ waveguides, and $\mathrm{f}_{R}$ will target the $10-100 \mathrm{GHz}$ range for large mode spacing in the comb. Absolute frequency measurement will depend on stabilising $\mathrm{f}_{C E O}$, which in turn relies on an octave spanning $\Delta \mathrm{f}$ and high comb output power.

\section{Conclusions}

The results presented here represent the first steps toward a compact platform for GHz frequency combs. The VECSELs the research community is able to grow and build, are fast approaching the performance needed to supply an entire generation of new devices, making easily resolvable frequency combs a reality. However, using a ML laser at PRFs near $1 \mathrm{GHz}$ requires significantly higher average powers to approach conventional ML lasers with PRF at sub-100 MHz. As such, materials with higher non-linear response and the possibility of microstructure design are of paramount importance. Here we have experimented with $\mathrm{Ta}_{2} \mathrm{O}_{5}$ waveguides to determine near-IR coupling and propagation characteristics, to eventually fabricate custom structures compatible with VECSEL wavelengths and peak powers. Strong doping in the target structures has highlighted that other processes can leech at the peak powers required for broadening and eventually coherent continuum generation. Un-doped structures however were easily capable of producing a broadened output, achieving over double the input breadth $(25$ $\mathrm{nm}-60 \mathrm{~nm}$ ) with sub-10 mm propagation through $\mathrm{Ta}_{2} \mathrm{O}_{5}$. This result coupled with $\mathrm{Ta}_{2} \mathrm{O}_{5}$ fabrication methods and opportunity for optimised design, gives a promising outlook to VECSEL continuum generation.

\section{ACKNOWLEDGMENTS}

The research was funded by the Engineering and Physical Sciences Research Council under the UK National Quantum Technologies Programme via the Hub in Sensors and Metrology. T. Chen Sverre is funded by an EPSRC research fellowship.

\section{REFERENCES}

[1] Udem, T., Holzwarth, R., and Hänsch, T. W., "Optical frequency metrology.," Nature 416(6877), 233-237 (2002).

[2] Eggleton, B. J., Moss, D. J., and Radic, S., [Optical Fiber Telecommunications V: Components and Subsystems], Academic Press, Oxford, UK (2008). 
[3] Bartels, A., , , Heinecke, D., and Diddams, S., "10-GHz Self-Referenced Optical Frequency Comb," Science 326(5953), 681 (2009).

[4] Kippenberg, T. J., Holzwarth, R., and Diddams, S. a., "Microresonator-based optical frequency combs.," Science (New York, N.Y.) 332, 555-9 (apr 2011).

[5] Klopp, P., Saas, F., Zorn, M., Weyers, M., and Griebner, U., "290-Fs Pulses From a Semiconductor Disk Laser.," Optics express 16(8), 5770-5775 (2008).

[6] Klopp, P., Griebner, U., Zorn, M., Klehr, A., Liero, A., Weyers, M., and Erbert, G., "Mode-locked InGaAsAlGaAs disk laser generating sub-200-fs pulses, pulse picking and amplification by a tapered diode amplifier.," Optics express 17(13), 10820-10834 (2009).

[7] Klopp, P., Griebner, U., Zorn, M., and Weyers, M., "Pulse repetition rate up to $92 \mathrm{GHz}$ or pulse duration shorter than 110 fs from a mode-locked semiconductor disk laser," Applied Physics Letters 98(7), 071103 (2011).

[8] Waldburger, D., Link, S. M., Mangold, M., Alfieri, C. G. E., Gini, E., Golling, M., Tilma, B. W., and Keller, U., "High-power 100 fs semiconductor disk lasers," Optica 3(8), 844-852 (2016).

[9] Wilcox, K. G., Mihoubi, Z., Elsmere, S., Quarterman, A., Foreman, H. D., Hashimoto, S., Su, T., Keller, U., and Tropper, A., "Passively modelocked $832 \mathrm{~nm}$ pulses at $1.9 \mathrm{GHz}$ repetition rate," 44(25), 4-5 (2008).

[10] Wilcox, K. G., Quarterman, A. H., Carswell, S., Mihoubi, Z., Apostolopoulos, V., Chung, A., and Tropper, A., "Mode-Locking Build-Up Measurements: Probing the Modelocking Mechanisms in Vertical-ExternalCavity Surface-Emitting Lasers," Conference on Lasers and Electro-Optics 2010 (2010).

[11] Wilcox, K. G., Quarterman, A. H., Beere, H., Ritchie, D. A., and Tropper, A. C., "High Peak Power Femtosecond Pulse Passively Mode-Locked Vertical-External-Cavity Surface-Emitting Laser," IEEE Photonics Technology Letters 22(14), 1021-1023 (2010).

[12] Wilcox, K. G., Quarterman, a. H., Beere, H. E., Ritchie, D. a., and Tropper, a. C., "Variable repetition frequency femtosecond-pulse surface emitting semiconductor laser," Applied Physics Letters 99(13), 131107 (2011).

[13] Wilcox, K. G., Quarterman, A. H., Beere, H. E., Ritchie, D. A., and Tropper, A. C., "Repetitionfrequency-tunable mode-locked surface emitting semiconductor laser between 2.78 and $7.87 \mathrm{GHz}$," Optics express 19(23), 23453-23459 (2011).

[14] Wilcox, K. G., Tropper, A. C., Beere, H. E., Ritchie, D. a., Heinen, B., and Stolz, W., "4.35 kW peak power femtosecond pulse mode-locked VECSEL for supercontinuum generation," Optics express 21(2), 1599-1605 (2013).

[15] Scheller, M., Wang, T.-L., Kunert, B., Stolz, W., Koch, S. W., and Moloney, J. V., "Passively modelocked VECSEL emitting 682 fs pulses with $5.1 \mathrm{~W}$ of average output power," Electronics Letters 48(10), 588 (2012).

[16] Lubeigt, W., Bialkowski, B., Lin, J., Head, C. R., Hempler, N., Maker, G. T., and Malcolm, G. P. A., "Commercial mode-locked vertical external cavity surface emitting lasers," Vertical External Cavity Surface Emitting Lasers (Vecsels) Vii 10087(February) (2017).

[17] Hoogland, S., Dhanjal, S., Tropper, A. C., Roberts, J. S., Häring, R., Paschotta, R., and Keller, U., "Passively Mode-Locked Diode-Pumped Surface-Emitting Semiconductor Laser," IEEE Photonics Technology Letters 12(9), 1135-1137 (2000).

[18] Gaafar, M., Nakdali, D. A., Möller, C., Fedorova, K. A., Wichmann, M., Shakfa, M. K., Zhang, F., Rahimiiman, A., Rafailov, E. U., and Koch, M., "Self-mode-locked quantum-dot vertical-external-cavity surfaceemitting laser," Optics letters 39(15), 4623-4626 (2014).

[19] Gaafar, M., Richter, P., Keskin, H., Möller, C., Wichmann, M., Stolz, W., Rahimi-Iman, A., and Koch, M., "Self-mode-locking semiconductor disk laser," Optics Express 22(23), 28390 (2014).

[20] Laurain, A., Marah, D., Rockmore, R., McInerney, J. G., Hader, J., Ruiz Perez, A., Koch, S. W., Stolz, W., and Moloney, J. V., "High power sub-200fs pulse generation from a colliding pulse modelocked VECSEL," 10087, 100870E (2017).

[21] Chen Sverre, T., Head, C., Turnbull, A., Shaw, E., Tropper, A., and Muskens, O., "Tunable repetition rate VECSEL for resonant acoustic-excitation of nanostructures," in [Proceedings of SPIE - The International Society for Optical Engineering], 9734 (2016). 
[22] Head, C. R., Chan, H.-y., Feehan, J. S., Shepherd, D. P., Alam, S.-u., Tropper, A. C., Price, J. H. V., and Wilcox, K. G., "Supercontinuum Generation With GHz Repetition Rate Femtosecond-Pulse Fiber-Amplified VECSELs," IEEE Photonics Technology Letters 25(5), 464-467 (2013).

[23] Head, C., Hein, A., Turnbull, A., Polanik, M., Shaw, E., Chen Sverre, T., Unger, P., and Tropper, A., "High-order dispersion in sub-200-fs pulsed VECSELs," in [Proceedings of SPIE - The International Society for Optical Engineering], 9734 (2016).

[24] Hoffmann, M., Sieber, O. D., Wittwer, V. J., Krestnikov, I. L., Livshits, D. A., Barbarin, Y., Südmeyer, T., and Keller, U., "Femtosecond high-power quantum dot vertical external cavity surface emitting laser," Optics Express 19(9), 8108 (2011).

[25] Zaugg, C. A., Sun, Z., Wittwer, V. J., Popa, D., Milana, S., Kulmala, T., Sundaram, R. S., Mangold, M., Sieber, O. D., Golling, M., Lee, Y., Ahn, J. H., Ferrari, A. C., and Keller, U., "Ultrafast and widely tuneable vertical-external-cavity surface-emitting laser, mode-locked by a graphene-integrated distributed Bragg reflector," 21(25), 739-747 (2013).

[26] Zaugg, C. A., Klenner, A., Mangold, M., Mayer, A. S., Link, S. M., Emaury, F., Golling, M., Gini, E., Saraceno, C. J., Tilma, B. W., and Keller, U., "Gigahertz self-referenceable frequency comb from a semiconductor disk laser," Optics Express 22(13), 16445-16455 (2014).

[27] Albrecht, A. R., Wang, Y., Ghasemkhani, M., Seletskiy, D. V., Cederberg, J. G., and Sheik-Bahae, M., "Exploring ultrafast negative Kerr effect for mode-locking vertical external-cavity surface-emitting lasers," Optics Express 21(23), 28801 (2013).

[28] Alfieri, C. G. E., Waldburger, D., Link, S. M., Gini, E., Golling, M., Tilma, B. W., Mangold, M., and Keller, U., "Recent progress in high-power ultrafast MIXSELs," Proc. of SPIE 9734, 973407 (2016).

[29] Mangold, M., Wittwer, V. J., Zaugg, C. A., Link, S. M., Golling, M., Tilma, B. W., and Keller, U., "Femtosecond pulses from a modelocked integrated external-cavity surface emitting laser (MIXSEL)," Optics Express 21(21), 24904 (2013).

[30] Mangold, M., Zaugg, C. a., Link, S. M., Golling, M., Tilma, B. W., and Keller, U., "Pulse repetition rate scaling from 5 to $100 \mathrm{GHz}$ with a high-power semiconductor disk laser," Optics Express 22(5), 6099 (2014).

[31] Mangold, M., Golling, M., Gini, E., Tilma, B. W., and Keller, U., "Sub-300-femtosecond operation from a MIXSEL," Optics Express 23(17), 22043 (2015).

[32] Sieber, O. D., Wittwer, V. J., Mangold, M., Hoffmann, M., Südmeyer, T., and Keller, U., "Femtosecond VECSEL with tunable multi-gigahertz repetition rate," Optics express 19(23), 23538-23543 (2011).

[33] Saarinen, E., Rantamaki, A., Chamorovskiy, A., and Okhotnikov, O., "200 GHz 1 W semiconductor disc laser emitting 800 fs pulses," Electronics Letters 48(21), 1355 (2012).

[34] Kahle, H., Mateo, C. M. N., Brauch, U., Bek, R., Schwarzbäck, T., Jetter, M., Graf, T., and Michler, P., "Gain chip design, power scaling and intra-cavity frequency doubling with LBO of optically pumped red-emitting AlGaInP-VECSELs," Proc. SPIE 9734 9734(0), 97340T-97340T-6 (2016).

[35] Kahle, H., Mateo, C. M. N., Brauch, U., Tatar-Mathes, P., Bek, R., Jetter, M., Graf, T., and Michler, P., "Semiconductor membrane external-cavity surface-emitting laser (MECSEL)," Optica 3(12), 1506 (2016).

[36] Kahle, H., Mateo, C. M. N., Brauch, U., Bek, R., Jetter, M., Graf, T., and Michler, P., "The optically pumped semiconductor membrane external-cavity surface-emitting laser (MECSEL): a concept based on a diamond-sandwiched active region," 10087(0), 100870J (2017).

[37] Subramanian, A. Z., Murugan, G. S., Zervas, M. N., and Wilkinson, J. S., "High index contrast Er:Ta2O5 waveguide amplifier on oxidised silicon," Optics Communications 285(2), 124-127 (2012).

[38] Rabiei, P., Rao, A., Chiles, J., Ma, J., and Fathpour, S., "Low-loss and high index-contrast tantalum pentoxide microring resonators and grating couplers on silicon substrates," Optics Letters 39(18), 5379 (2014).

[39] Rabiei, P., Ma, J., Khan, S., Chiles, J., and Fathpour, S., "Micro-ring optical resonators fabricated by selective oxidation of refractory metals (SORM)," Optical Fiber Communication Conference/National Fiber Optic Engineers Conference 2013, OTu3C.5 (2013).

[40] Rabiei, P., Ma, J., Khan, S., Chiles, J., and Fathpour, S., "Submicron optical waveguides and microring resonators fabricated by selective oxidation of tantalum.," Optics express 21(6), 6967-72 (2013). 
[41] Jiang, X., Joly, N. Y., Finger, M. a., Babic, F., WongGordon, K. L., Travers, J. C., and RussellPhilip St, J., "Deep-ultraviolet to mid-infrared supercontinuum generated in solid-core ZBLAN photonic crystal fibre," Nature Photonics 9(2), 133-139 (2015).

[42] Subramanian, A. Z., Oton, C. J., Shepherd, D. P., and Wilkinson, J. S., "Erbium-doped waveguide laser in tantalum pentoxide," IEEE Photonics Technology Letters 22(21), 1571-1573 (2010).

[43] Singh, M. K., Fusegi, G., Kano, K., Bange, J. P., Miura, K., and Hanaizumi, O., "Intense photoluminescence from erbium-doped tantalum oxide thin films deposited by sputtering," IEICE Electronics Express 6(23), 1676-1682 (2009).

[44] Huang, S. W., Liu, H., Yang, J., Yu, M., Kwong, D. L., and Wong, C. W., "Smooth and flat phase-locked Kerr frequency comb generation by higher order mode suppression," Nature Scientific Reports 6(February), $1-7(2016)$. 